\title{
sciendo
}

Transport and Telecommunication, 2021, volume 22, no. 3, 353-366

Transport and Telecommunication Institute, Lomonosova 1, Riga, LV-1019, Latvia

DOI $10.2478 /$ ttj-2021-0028

\section{THE METHOD OF DETERMINING THE OPTIMAL TRANSMISSION RESOURCES DISTRIBUTION OF ICT NETWORKS MODELLED BY GRAPHS}

\author{
Stawomir Bujnowski ${ }^{1}$, Beata Marciniak ${ }^{2}$, Tomasz Marciniak ${ }^{3}$, Zbigniew Lutowski $^{4}$ \\ ${ }^{I}$ UTP University of Science and Technology, Faculty of Telecommunication, Information Technology and \\ Electrical Engineering, Al.prof. S. Kaliskiego 7, 85-796 Bydgoszcz, Poland \\ ${ }^{1}$ Slawomir.Bujnowski@utp.edu.pl, ${ }^{2}$ Beata.Marciniak@utp.edu.pl, ${ }^{3}$ Tomasz.Marciniak@utp.edu.pl \\ ${ }^{4}$ Zbigniew.Lutowski@utp.edu.pl
}

\begin{abstract}
The paper discusses the method of improvement of transmission properties of ICT networks by setting the correct distribution of transmission resources in order that it is possible to increase the resulting global probability of supporting the calls generated in the nodes included in the network. The method uses some parts of the graph theory. A minimum length path searching algorithm was used which made it possible to calculate the volume of movement transferred by individual edges of the graph describing the network. The coefficient of the distribution of transmission resources was determined and used to calculate the value of the resources in reference to a particular link, both in the case of equal and diverse movement generated in individual network nodes. A piece of software was developed which uses the results of applying the above-mentioned algorithm to prepare the files describing the analyzed structures in order to carry out simulation tests. The views and analyses are illustrated by examples, and the resulting effects of the influence of the introduction of the method of dividing network resources into transmission properties of a network modelled by graphs are shown in the charts included.
\end{abstract}

Keywords: telecommunication network, graph, simulation

\section{Introduction}

The primary purpose of the theory of the information and communications technology used for the transmission of data, video and audio is the construction of analytical models of networks and ICT systems. It is crucial for their proper design and management. Its expected result is to obtain the best possible effect with given resources or the minimization of the resources usage while reaching the assumed goal. To this end the theory of probability, algebra, the theory of Markov processes and the graph theory are used among other things.

Network resource management is the process of adjusting resources of a network, such as bandwidth, storage, etc., with the purpose of improving the performance of the network. It is very important, especially for WSN networks (Wireless Sensor Network). The unique characteristic of WSN network poses several challenges in their design. These include limited battery capacity, limited hardware and resources, massive and random deployment, dynamically changing and unreliable environment. Sensor nodes in WSN networks are strictly limited in terms of transmission power, onboard energy, processing and storage capacity, and therefore require careful resource management. Recent research of the WSN network has led to the creation of many new protocols designed specifically for sensor networks in which energy saving is an important factor. In the literature, the most emphasis is put on routing protocols, as they may be various depending on the application and the network architecture (Heinzelman et al., 2002), (Kaur et al., 2015), (Mansourkiaie et al., 2016). Their goal is to find ways to combine energy-saving routes and reliably transfer data from sensor nodes to the base station in the core of network layer, so that the network lifetime is maximized.

The problem of resource management in networks is also widely discussed in optical networks, Software Defined Network (SDN) and Network Virtualization process. Elastic optical networks (EON) are a promising technology because they feature high-capacity and dynamic bandwidth, which are in demand in the newest wireless networks. However, they are not flawless. This brought attention of Wang et al. (2019) who analysed EON state and proposed a novel method of load-aware nonlinearity estimation. They focused on effective resource distribution for networks to minimize the resource use while maintaining the required bandwidth and high quality of transmission. These solutions were 
measured in a simulation. Obtained results showed that this model can be successfully used to minimize the amount of allocated frequency slots while improving network capacity at the same time. Elastic optical networks are also used to correspond to rapid growth of Internet traffic. Those networks are empowered by multirate transmission and bandwidth-variable switching technology. Routing and Spectrum Allocation (RSA) is one of essential elements in realizing EONs. In this paper authors (Chen et al., 2015) focus on achieving better RSA performance with reduced computational complexity. Authors (Tanaka et al., 2016) also focus on using EONs to optimize optical layer traffic. This paper proposes an algorithm of multilayer network reconfiguration which supports periodically changing IP traffic patterns. Authors proposed two solution schemes: first scheme consists in bandwidth resizing achieved through subcarrier expansion and reduction, and the second uses energy-efficient adaptive modulation according to the data rate and distance of the client demands.

In the paper (Liu et al., 2015) authors focus on controlling resources. To allow realization of network programmability and application-aware networking SDN architecture should be based on separation of control and data plane in network devices. The importance of mapping applications to network resources is stressed as well as maintaining alike mapping in the resource management of the SDN controller. In this paper authors proposed the mechanism of mapping and its adequate implementation.

In paper (Li et al., 2017) authors focused on the fact that virtual networks share the same physical network. However, the resources of the physical network are limited. Therefore, it is necessary to improve their utilization. Since the resource requirements may change in time, the authors proposed a novel mechanism based on predictions to sustain optimal resource usage. Predictions were compared with real life requirements to ensure their reliability. This mechanism was created to allow dynamic resources allocation to the virtual networks. Obtained results proved that this approach can increase the utilization of the physical resource. Their conclusion was that the prediction-based mechanism improves the acceptance ratio of virtual network, but also ensures the requirement of virtual networks. They announced further work on the algorithm to prove its usefulness in practice.

In papers (Dai et al., 2014), (Ramaswami et al., 2010) authors used seamless networking scheme for Fiber Wireless networks based on virtualization. Hierarchical model, service model, service implementation and dynamic bandwidth assignment were used as well. In the next step authors evaluated how network changes before and after visualization. In the proposed network scheme they achieved better performance in comparison to the traditional networking scheme.

ICT systems must satisfy the increasing requirements of quality, speed and reliability of information transmission (Coffman et al., 2002). In order to make it possible network nodes are fitted with state-of-the-art devices. The structure of connections between these nodes is also important (Bhuyan et al., 1987), (Pedersen et al., 2008), (Xu, 2010). ICT systems are often described with graphs (Deswal et al., 2012), (Kotsis, 1992) with vertices constituted by nodes with exchange functions and edges formed most frequently with bidirectional, independent transmission channels.

\section{The work}

Article (Diestel, 2001) discusses the results of the analysis of the influence of the number of uses of individual edges of the graphs describing the ICT networks and eventuated in offering a way to control resources of transmission channels which as a result will make it possible to improve the transmission properties of these networks. The term 'transmission properties of a network' means the probability of rejecting the service call for $\mathrm{P}_{\text {rej }}$ of the traffic generated by the users in the nodes constituting the network, and 'resources' means for example the bandwidth used for transmitting data between nodes.

Simulation tests were performed for the virtual networks modelled by graphs, both regular and irregular. The authors have described it in their publications (Ledziński et al., 2017), (Bujnowski et al., 2017), (Bujnowski et al., 2019), (Bujnowski et al., 2018). The tests were intended to compare the abovementioned parameter in virtual networks with and without the resource control.

The assumption during the tests was that the traffic generated in each node is equally distributed between the remaining nodes which is not true. Because of this another approach to the issue was proposed which is the subject of this paper.

In order to explain the rules of the proposed way of conduct an example of a simple network described with the graph in Figure 1 was used. 


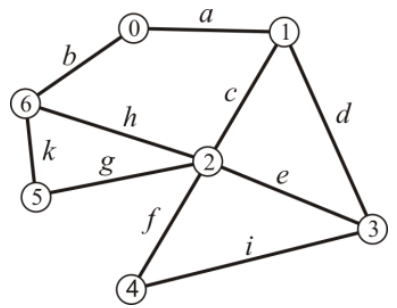

Figure 1. The graph describing the analysed network

The structure of the connections in the analysed network can be described with an adjacency matrix $\left[M_{S}\right]$ :

$\left[M_{s}\right]=\left[\begin{array}{lllllll}0 & 1 & 0 & 0 & 0 & 0 & 1 \\ 1 & 0 & 1 & 1 & 0 & 0 & 0 \\ 0 & 1 & 0 & 1 & 1 & 1 & 1 \\ 0 & 1 & 1 & 0 & 1 & 0 & 0 \\ 0 & 0 & 1 & 1 & 0 & 0 & 0 \\ 0 & 0 & 1 & 0 & 0 & 0 & 1 \\ 1 & 0 & 1 & 0 & 0 & 1 & 0\end{array}\right]$.

The matrix $\left[M_{S}\right]$ was transformed using the name given in Figure 1 into a transformed matrix $\left[M_{S T}\right]$, which also describes the structure of the analysed network.

$\left[M_{S T}\right]=\left[\begin{array}{lllllll}0 & a & 0 & 0 & 0 & 0 & b \\ a & 0 & c & d & 0 & 0 & 0 \\ 0 & c & 0 & e & f & g & h \\ 0 & d & e & 0 & i & 0 & 0 \\ 0 & 0 & f & i & 0 & 0 & 0 \\ 0 & 0 & g & 0 & 0 & 0 & k \\ b & 0 & h & 0 & 0 & k & 0\end{array}\right]$.

By using the minimum length paths searching algorithm a set of the paths was determined (a minimum length path is a path connecting nodes $v_{i} \mathrm{i} v_{j}$ which consists of the smallest number of edges).

The operating principle of this algorithm is the exponentiation of the adjacency matrices $\left[M_{S T}\right]$ (the power of a matrix is equal to the number of edges creating a path (Graham et al., 1994) and preparing tables presenting the connection system between each node and the remaining elements of the structure. The operation is active until it is established that all nodes are interconnected (it is assumed that the network is described with a consistent graph).

Table 1 shows the set of minimum length paths created with two edges. The table does not include the elements corresponding to the connection of the node with itself.

Table 1. The structures if minimum length paths created with two edges

\begin{tabular}{|c|c|c|c|c|c|c|c|}
\hline Node & 0 & 1 & 2 & 3 & 4 & 5 & 6 \\
\hline 0 & - & 0 & $a c, b h$ & $a d$ & 0 & $b k$ & 0 \\
\hline 1 & 0 & - & 0 & 0 & $c f, d i$ & $c g$ & $a b, c h$ \\
\hline 2 & $c a, h b$ & 0 & - & 0 & 0 & 0 & 0 \\
\hline 3 & $d a$ & 0 & 0 & - & 0 & $e g$ & $e h$ \\
\hline 4 & 0 & $f c, i d$ & 0 & 0 & - & $f g$ & $f h$ \\
\hline 5 & $k b$ & $g c$ & 0 & $g e$ & $g f$ & - & 0 \\
\hline 6 & 0 & $b a, h c$ & 0 & he & $h f$ & 0 & - \\
\hline
\end{tabular}

The analysis of the matrix $\left[M_{S T}\right]$ and the above table shows that the path connecting the zero and the fourth node is missing. It was established that the two nodes are connected with three parallel paths with three edges with acf, adi and $b h f$ configurations.

When all nodes in the network are interconnected, a table with results is built which includes all the elements corresponding to the shortest paths connecting individual recipient nodes with the source node.

Table 2 presents the set of minimum length paths in the analysed structure. 
Table 2. The set of minimum length paths

\begin{tabular}{|c|c|c|c|c|c|c|c|}
\hline Node & 0 & 1 & 2 & 3 & 4 & 5 & 6 \\
\hline 0 & - & $a$ & $a c, b h$ & $a d$ & $\begin{array}{c}a c f, a d i, \\
b h f\end{array}$ & $b k$ \\
\hline 1 & $a$ & - & $c$ & $d$ & $c f, d i$ & $c g$ & $a b, c h$ \\
\hline 2 & $c a, h b$ & $c$ & - & $e$ & $f$ & $g$ & $h$ \\
\hline 3 & $d a$ & $d$ & $e$ & - & $i$ & $e g$ & $e h$ \\
\hline 4 & $a c f, a d i$, & $f c, i d$ & $f$ & $i$ & - & $f g$ \\
\hline 5 & $k b$ & $g c$ & $g$ & $g e$ & $g f$ & - & $k$ \\
\hline 6 & $b$ & $b a, h c$ & $h$ & $h e$ & $h f$ & $k$ & - \\
\hline
\end{tabular}

In the analysed example it was assumed that the traffic generated between the nodes is distributed as shown in Table 3.

Table 3. The distribution of the traffic generated by the nodes

\begin{tabular}{|l|l|l|l|l|l|l|l|}
\hline Node & 0 & 1 & 2 & 3 & 4 & 5 & 6 \\
\hline 0 & - & 0.20 & 0.40 & 0.10 & 0.05 & 0.10 & 0.15 \\
\hline 1 & 0.15 & - & 0.45 & 0.15 & 0.05 & 0.10 & 0.10 \\
\hline 2 & 0.05 & 0.30 & - & 0.25 & 0.2 & 0.05 & 0.15 \\
\hline 3 & 0.15 & 0.20 & 0.50 & - & 0.05 & 0.05 & 0.05 \\
\hline 4 & 0.05 & 0.10 & 0.50 & 0.15 & - & 0.10 & 0.10 \\
\hline 5 & 0.10 & 0.05 & 0.35 & 0.05 & 0.10 & - & 0.10 \\
\hline 6 & 0.20 & 0.05 & 0.40 & 0.10 & 0.15 & 0.10 & - \\
\hline
\end{tabular}

The row cells above diagonal of the table 3 define the distribution of the traffic directed from a chosen source node to the recipient nodes, and the elements below the diagonal - to the volume of traffic directed to the source node.

To illustrate the further procedure, Table 4 shows the results of the calculations presenting the usage of individual edges in every possible minimum length path connecting any two nodes of the analysed network including their usage in the assumed traffic distribution.

Table 4. The results of the calculations of the traffic transmitted through individual edges

\begin{tabular}{|c|c|c|c|c|c|c|c|c|}
\hline$l=0$ & 0 & 1 & 2 & 3 & 4 & 5 & 6 & $H_{l}$ \\
\hline$a$ & & 0.2 & \begin{tabular}{|l|}
0.2 \\
\end{tabular} & 0.1 & 0.033 & & & 0,533 \\
\hline$b$ & & & \begin{tabular}{|l|}
0.2 \\
\end{tabular} & & 0.017 & 0.1 & 0.15 & 0,467 \\
\hline$c$ & & & 0.2 & & 0.017 & & & 0,217 \\
\hline$d$ & & & & 0.1 & 0.017 & & & 0,117 \\
\hline$e$ & & & & & & & & 0,000 \\
\hline$f$ & & & & & 0.033 & & & 0,033 \\
\hline$g$ & & & & & & & & 0,000 \\
\hline$h$ & & & 0.2 & & 0.017 & & & 0,217 \\
\hline$i$ & & & & & 0.017 & & & 0,017 \\
\hline$k$ & & & & & 0.033 & 0.1 & & 0,100 \\
\hline$l=1$ & 0 & 1 & 2 & 3 & 4 & 5 & 6 & $H_{l}$ \\
\hline$a$ & 0.15 & & & & & & 0.05 & 0,200 \\
\hline$b$ & & & & & & & 0.05 & 0,050 \\
\hline$c$ & & & 0.45 & & 0.025 & 0.1 & 0.05 & 0,625 \\
\hline$d$ & & & & 0.15 & 0.025 & & & 0,175 \\
\hline$e$ & & & & & & & & 0,000 \\
\hline$f$ & & & & & 0.025 & & & 0,025 \\
\hline$g$ & & & & & & 0.1 & & 0,100 \\
\hline$h$ & & & & & & & 0.05 & 0,050 \\
\hline$i$ & & & & & 0.025 & & & 0,025 \\
\hline$k$ & & & & & & & & 0,000 \\
\hline$l=2$ & 0 & 1 & \begin{tabular}{|l|}
2 \\
\end{tabular} & 3 & 4 & 5 & 6 & $H_{l}$ \\
\hline$a$ & 0.025 & & & & & & & 0,025 \\
\hline$b$ & 0.025 & & & & & & & 0,025 \\
\hline$c$ & 0.025 & 0.3 & & & & & & 0,325 \\
\hline$d$ & & & & & & & & 0,000 \\
\hline$e$ & & & & 0.25 & & & & 0,250 \\
\hline$f$ & & & & & 0.2 & & & 0,200 \\
\hline$g$ & & & & & & 0.05 & & 0,050 \\
\hline
\end{tabular}


Continuation of Table 4

\begin{tabular}{|c|c|c|c|c|c|c|c|c|}
\hline$h$ & 0.025 & & & & & & 0.15 & 0,175 \\
\hline $\bar{i}$ & & & & & & & & 0,000 \\
\hline$k$ & & & & & & & & 0,000 \\
\hline$l=3$ & 0 & 1 & 2 & 3 & 4 & 5 & 6 & $H_{l}$ \\
\hline$a$ & 0.15 & & & & & & & 0,15 \\
\hline$b$ & & & & & & & & 0,00 \\
\hline$c$ & & & & & & & & 0,00 \\
\hline$d$ & 0.15 & 0.2 & & & & & & 0,35 \\
\hline$e$ & & & 0.5 & & & 0.05 & 0.05 & 0,60 \\
\hline$f$ & & & & & & & & 0,00 \\
\hline$g$ & & & & & & 0.05 & & 0,05 \\
\hline$h$ & & & & & & & 0.05 & 0,05 \\
\hline$i$ & & & & & 0.05 & & & 0,05 \\
\hline$k$ & & & & & & & & 0,00 \\
\hline$l=4$ & 0 & 1 & 2 & 3 & 4 & 5 & 6 & $H_{l}$ \\
\hline$a$ & 0.033 & & & & & & & 0,033 \\
\hline$b$ & 0.017 & & & & & & & 0,017 \\
\hline$c$ & 0.017 & 0.05 & & & & & & 0,067 \\
\hline$d$ & 0.017 & 0.05 & & & & & & 0,067 \\
\hline$e$ & & & & & & & & 0,000 \\
\hline$f$ & 0.033 & 0.05 & 0.5 & & & 0.1 & 0.1 & 0,783 \\
\hline$g$ & & & & & & 0.1 & & 0,100 \\
\hline$h$ & 0.017 & & & & & & 0.1 & 0,117 \\
\hline $\bar{i}$ & 0.017 & 0.05 & & 0.15 & & & & 0,217 \\
\hline$k$ & & & & & & & & 0,000 \\
\hline$l=5$ & 0 & 1 & 2 & 3 & 4 & 5 & 6 & $H_{l}$ \\
\hline$a$ & & & & & & & & 0,00 \\
\hline$b$ & 0.1 & & & & & & & 0,10 \\
\hline$c$ & & 0.05 & & & & & & 0,05 \\
\hline$d$ & & & & & & & & 0,00 \\
\hline$e$ & & & & 0.05 & & & & 0,05 \\
\hline$f$ & & & & & 0.1 & & & 0,10 \\
\hline$g$ & & 0.05 & 0.35 & 0.05 & 0.1 & & & 0,55 \\
\hline$h$ & & & & & & & & 0,00 \\
\hline$i$ & & & & & & & & 0,00 \\
\hline$k$ & 0.1 & & & & & & 0.35 & 0,45 \\
\hline$l=6$ & 0 & 1 & 2 & 3 & 4 & 5 & 6 & $H_{l}$ \\
\hline$a$ & & 0.025 & & & & & & 0,025 \\
\hline$b$ & 0.2 & 0.025 & & & & & & 0,225 \\
\hline$c$ & & 0.025 & & & & & & 0,025 \\
\hline$d$ & & & & & & & & 0,000 \\
\hline$e$ & & & & 0.1 & & & & 0,100 \\
\hline$f$ & & & & & 0.15 & & & 0,150 \\
\hline$g$ & & & & & & & & 0,000 \\
\hline$h$ & & 0.025 & 0.4 & 0.1 & 0.15 & & & 0,675 \\
\hline$i$ & & & & & & & & 0,000 \\
\hline$k$ & & & & & & 0.1 & & 0,100 \\
\hline
\end{tabular}

The $H_{l}$ is the total traffic transmitted through a given edge in both directions, $l$ - node number. The procedure used in building Table 4 is as follows.

If the nodes are interconnected with:

- one or more subsequent edges forming a minimum length path, the element of the table corresponding to the given edge includes the value from the table presenting the distribution of traffic generated by the node.

For example, if edge $a$ was analysed, the value of connection $0-1$ would be 0.2 , the value of connection 1 - 0 would be 0.15 and for path $0-3$ or $3-0(a d)-0.1$;

- two or more parallel paths consisting of any number of edges. The table includes the value of the cell corresponding to these connections divided by the number of the parallel paths.

For example: The parallel paths connecting the nodes $0-2$ and $2-0$ consist of edge $a c$ and $b h$. In both cases the value for edge $a$ is 0.2 . There are three paths between nodes $0-4$ and $4-0$. They are made up of edges acf, adi, bhf. Both cells of the table include the value $0.05 / 3 * 2=0.0333$ since it is necessary to take into account the presence of edge $a$ into paths. 
The sum of the results for every edge gives the values of usage of the edges in the analysed network (Table 5).

Table 5. Parts of the traffic transmitted through individual edges

\begin{tabular}{|c|c|c|c|c|c|c|}
\hline Edge & $a$ & $b$ & $c$ & $d$ & $e$ & \\
\hline$\sum_{n=1}^{N} H_{l n}$ & 0.967 & 0.883 & 1.308 & 0.708 & 1.000 & \\
\hline Edge & $f$ & $\mathrm{~g}$ & $\mathrm{~h}$ & $\mathrm{i}$ & $\mathrm{k}$ & $\sum \mathrm{H}$ \\
\hline$\sum_{n=1}^{N} H_{l n}$ & 1.292 & 0.850 & 1.283 & 0.308 & 0.650 & 9.250 \\
\hline
\end{tabular}

where: $\sum H_{l n}$ means the part of the intermodal traffic transmitted through the given edge in both directions, $\sum H$ - total traffic, $N$ - the number of nodes.

The presented information shows that the least loaded edge is $i$, and the most - edges $c, f$ and $h$.

To verify the influence of the used method of the distribution of resources on the transmission properties of the analysed networks, a coefficient $w_{d r c}$ of the distribution of transmission resources was introduced which can be calculated from the following formula:

$w_{d r c l}=\sum_{n=1}^{N} H_{l n} \frac{1}{\sum H}$.

The resources are divided according to the following rule:

$R E S_{l}=w_{d r c n} \cdot R E S_{g}$

where $R E S_{l}$ is the resources used by the $l$-th edge and $R E S_{g}$ is the total network resources.

Table 6 includes the distribution of resources for the analysed example with the assumption that the number global resources which can be used is 320 conventional transmission units.

Table 6. The calculated resource distribution

\begin{tabular}{|l|l|l|l|l|l|}
\hline Edge & $\mathrm{a}$ & $\mathrm{b}$ & $\mathrm{c}$ & $\mathrm{d}$ & $\mathrm{e}$ \\
\hline$w_{\text {drcl }}$ & 0.105 & 0.095 & 0.141 & 0.077 & 0.108 \\
\hline RES & 34 & 30 & 45 & 25 & 35 \\
\hline Edge & $\mathrm{f}$ & $\mathrm{g}$ & $\mathrm{h}$ & $\mathrm{i}$ & $\mathrm{k}$ \\
\hline$w_{\text {drcl }}$ & 0.140 & 0.092 & 0.139 & 0.033 & 0.070 \\
\hline$R E S_{l}$ & 45 & 29 & 44 & 11 & 22 \\
\hline
\end{tabular}

The above calculation results are valid for the situation in which every node generates the same traffic intensity and only the diversified distribution of the traffic between target nodes is considered. To make the research more realistic, the possibility to control the volume of traffic in each node was incorporated (the method is extensively described in the paper [19]). It consists in multiplying the transformed matrix $\left[M_{S T}\right]$ by the traffic matrix $\left[M_{T}\right]$ which includes the values of the traffic generated in reference to any chosen node.

To illustrate the procedure the analysed example was used again.

It was assumed that the intensity of traffic in nodes 1 and 3 is two times larger and in node 6 three times larger than in other nodes. Matrix $\left[M_{T}\right]$ will be structured as follows:

$\left[M_{T}\right]=\left[\begin{array}{lllllll}0 & 2 & 1 & 2 & 1 & 1 & 3 \\ 1 & 0 & 1 & 2 & 1 & 1 & 3 \\ 1 & 2 & 0 & 2 & 1 & 1 & 3 \\ 1 & 2 & 1 & 0 & 1 & 1 & 3 \\ 1 & 2 & 1 & 2 & 0 & 1 & 3 \\ 1 & 2 & 1 & 2 & 1 & 0 & 3 \\ 1 & 2 & 1 & 2 & 1 & 1 & 0\end{array}\right]$.

Using the method described earlier Table 7 was created which describes the configuration of minimum length paths connecting the nodes of the network. 
Table 7. The configuration of minimum length paths used for the transmission of information in unequal distribution of the generated traffic

\begin{tabular}{|c|c|c|c|c|c|c|c|}
\hline Node & 0 & 1 & 2 & 3 & 4 & 5 & 6 \\
\hline 0 & - & $a$ & $a c, b h$ & $a d$ & acf,adi,bhf & $b k$ & $b$ \\
\hline 1 & $2 a$ & - & $2 c$ & $2 d$ & $2 c f, 2 d i$ & $2 c g$ & $2 a b, 2 c h$ \\
\hline 2 & $c a, h b$ & $c$ & - & $e$ & $f$ & $g$ & $h$ \\
\hline 3 & $2 d a$ & $2 d$ & $2 e$ & - & $i$ & $2 e g$ & $2 e h$ \\
\hline 4 & acf,adi,bhf & $f_{c, i d}$ & $f$ & $i$ & - & $f g$ & $f h$ \\
\hline 5 & $k b$ & $g c$ & $g$ & $g e$ & $g f$ & - & $k$ \\
\hline 6 & $3 b$ & $3 b a, 3 h c$ & $3 h$ & $3 h e$ & $3 h f$ & $3 k$ & - \\
\hline
\end{tabular}

Table 8 contains values which present the usage of individual edges in the analysed case and the values calculated for $w_{d r c l}$ coefficients as well as the distribution of resources $\left(R E S_{g}=320\right)$ made in accordance with the rule (2).

Table 8. Distribution of resources in case of diverse traffic generated in nodes

\begin{tabular}{|c|c|c|c|c|c|}
\hline Edge & $\mathrm{a}$ & $\mathrm{b}$ & $\mathrm{c}$ & $\mathrm{d}$ & $\mathrm{e}$ \\
\hline$\sum_{n=1}^{N} H_{l n}$ & 1.367 & 1.383 & 1.983 & 1.233 & 1.800 \\
\hline$w_{d r c l}$ & 0.095 & 0.096 & 0.138 & 0.086 & 0.125 \\
\hline$R E S_{l}$ & 30 & 31 & 44 & 28 & 40 \\
\hline Edge & $\mathrm{f}$ & $\mathrm{g}$ & $\mathrm{h}$ & $\mathrm{i}$ & $\mathrm{k}$ \\
\hline$\sum_{n=1}^{N} H_{l n}$ & 1.617 & 1.000 & 2.733 & 0.383 & 0.850 \\
\hline$w_{d r c l}$ & 0.113 & 0.070 & 0.190 & 0.027 & 0.059 \\
\hline$R E S_{l}$ & 36 & 22 & 61 & 9 & 19 \\
\hline
\end{tabular}

The values obtained this way include the unevenness of the traffic generated in network nodes.

The given procedure is the operating principle of an auxiliary software which was used to prepare data for the simulator used to check the influence of resource control on transmission properties of network modelled by graphs.

The developed software works according to the following algorithm.

A table is created which describes the percentage of traffic directed to individual nodes of the network (in this example Table 3 illustrates the procedure).

After loading the table, the developed software calculates the values of the determined parameters: the total value of the traffic transmitted through the edges, the values of $w_{d r c l}$ coefficients and the distribution of resources for individual edges.

Table 9. The total value of traffic transmitted through edges

\begin{tabular}{|l|l|l|l|l|l|l|l|}
\hline Node[0]-> & 0.00 & 1.37 & 0.00 & 0.00 & 0.00 & 0.00 & 1.38 \\
\hline Node[1]-> & 1.37 & 0.00 & 1.98 & 1.23 & 0.00 & 0.00 & 0.00 \\
\hline Node[2]-> & 0.00 & 1.98 & 0.00 & 1.80 & 1.62 & 1.00 & 2.73 \\
\hline Node[3]-> & 0.00 & 1.23 & 1.80 & 0.00 & 0.38 & 0.00 & 0.00 \\
\hline Node[4]-> & 0.00 & 0.00 & 1.62 & 0.38 & 0.00 & 0.00 & 0.00 \\
\hline Node[5]-> & 0.00 & 0.00 & 1.00 & 0.00 & 0.00 & 0.00 & 0.85 \\
\hline Node[6]-> & 1.38 & 0,00 & 2.73 & 0.00 & 0.00 & 0.85 & 0.00 \\
\hline
\end{tabular}

Table 10. The $w_{d r c l}$ coefficient

\begin{tabular}{|l|l|l|l|l|l|l|l|}
\hline wdrc[0]-> & 0.000 & 0.095 & 0.000 & 0.000 & 0.000 & 0.000 & 0.096 \\
\hline wdrc [1]-> & 0.095 & 0.000 & 0.138 & 0.086 & 0.000 & 0.000 & 0.000 \\
\hline wdrc [2]-> & 0.000 & 0.138 & 0.000 & 0.125 & 0.113 & 0.700 & 0.190 \\
\hline wdrc [3]-> & 0.000 & 0.086 & 0.125 & 0.000 & 0.027 & 0.000 & 0.000 \\
\hline wdrc [4]-> & 0.000 & 0.000 & 0.113 & 0.027 & 0.000 & 0.000 & 0.000 \\
\hline wdrc [5]-> & 0.000 & 0.000 & 0.700 & 0.000 & 0.000 & 0.000 & 0.059 \\
\hline wdrc [6]-> & 0.096 & 0,000 & 0.190 & 0.000 & 0.000 & 0.059 & 0.000 \\
\hline
\end{tabular}


Table 11. The distribution of resources between individual edges

\begin{tabular}{|l|l|l|l|l|l|l|l|}
\hline $\mathrm{RES}_{1}[0]->$ & 0 & 30 & 0 & 0 & 0 & 0 & 31 \\
\hline $\mathrm{RES}_{1}[1]->$ & 30 & 0 & 44 & 28 & 0 & 0 & 0 \\
\hline $\mathrm{RES}_{1}[2]->$ & 0 & 44 & 0 & 40 & 36 & 22 & 61 \\
\hline $\operatorname{RES}_{1}[3]->$ & 0 & 28 & 40 & 0 & 9 & 0 & 0 \\
\hline $\operatorname{RES}_{1}[4]->$ & 0 & 0 & 36 & 9 & 0 & 0 & 0 \\
\hline $\operatorname{RES}_{1}[5]->$ & 0 & 0 & 22 & 0 & 0 & 0 & 19 \\
\hline $\operatorname{RES}_{1}[6]->$ & 31 & 0 & 61 & 0 & 0 & 19 & 0 \\
\hline
\end{tabular}

On the basis of the results the software creates the following text file used for simulation.

$$
\begin{aligned}
& 0,00 ; 1,30,20 ; 0,40 ; 0,10 ; 0,50 ; 0,10 ; 1,31,15 ; \mathrm{X}, 1 \\
& 1,30,15 ; 0,00 ; 1,44,45 ; 1,28,15 ; 0,50 ; 0,10 ; 0,10 ; \mathrm{X}, 2 \\
& 0,50 ; 1,44,30 ; 0,00 ; 1,40,25 ; 1,36,20 ; 1,22,5 ; 1,61,15 ; \mathrm{X}, 1 \\
& 0,15 ; 1,28,20 ; 1,40,50 ; 0,00 ; 1,9,5 ; 0,50 ; 0,50 ; \mathrm{X}, 2 \\
& 0,50 ; 0,10 ; 1,36,50 ; 1,9,15 ; 0,00 ; 0,10 ; 0,10 ; \mathrm{X}, 1 \\
& 0,10 ; 0,50 ; 1,22,35 ; 0,50 ; 0,10 ; 0,00 ; 1,19,35 ; \mathrm{X}, 1 \\
& 1,31,20 ; 0,50 ; 1,61,40 ; 0,10 ; 0,15 ; 1,19,10 ; 0,00 ; \mathrm{X}, 3
\end{aligned}
$$

If the file element has the form:

- $\quad 0, p$, it means that $p$ percent of generated traffic is directed from the source node to the target node, the path connecting the nodes has at least two edges;

- $\quad 1, r, p$ corresponds to the edge directly connecting two edges, $r$ - resources assigned do the edge, $p$ - the percentage of the generated traffic;

- $\mathrm{X}, v$ - the volume of traffic generated in a given source node, $v$ - a coefficient defining the amount of traffic in relation to the node generating the leash volume of traffic. For example, in the given node 1 the traffic is two times larger than in node 0 .

Below is an example of a print of results of a test for the network shown in Figure 1 including the adjustment of resources and different volume of traffic generated in individual network nodes.

$$
\begin{aligned}
& \text { 2019-12-09 22:08:35 START } \\
& \begin{array}{llll}
\text { Traffic } 10 & \mathrm{OK}=3998557 & \mathrm{BAD}=1443 & 0.00036075 \\
\text { Traffic } 15 & \mathrm{OK}=3962395 & \mathrm{BAD}=37605 & 0,00940125 \\
\text { Traffic } 20 & \mathrm{OK}=3698967 & \mathrm{BAD}=301033 & 0,07525825 \\
\text { Traffic } 25 & \mathrm{OK}=3247008 & \mathrm{BAD}=752992 & 0,188248 \\
\text { Traffic } 30 & \mathrm{OK}=2837116 & \mathrm{BAD}=1162884 & 0,290721 \\
\text { Traffic } 35 & \mathrm{OK}=2504377 & \mathrm{BAD}=1495623 & 0,37390575 \\
\text { Traffic } 40 & \mathrm{OK}=2238496 & \mathrm{BAD}=1761504 & 0,440376 \\
\text { Traffic } 45 & \mathrm{OK}=2023065 & \mathrm{BAD}=1976935 & 0,49423375 \\
\text { Traffic } 50 & \mathrm{OK}=1847364 & \mathrm{BAD}=2152636 & 0,538159
\end{array}
\end{aligned}
$$

\section{9-12-09 22:08:54 END}

The traffic generated in the nodes kept changing within the range of 10-50 [Erl] while included were the values of coefficients $v$ assigned to a given node, OK means the number of served calls, BAD is the number of unserved calls. The last column contains the values of global probability of the realization of a call.

By using the above tool the tests of the analysed structure were carried out for the cases with and without the control of transmission resources with the same and diversified volume of traffic generated in the nodes. The result are shown in charts (Figures 2, 3). 


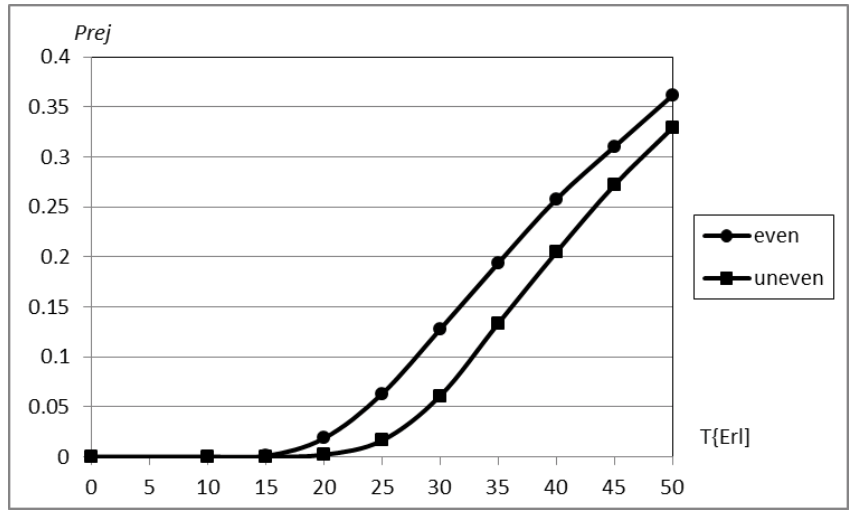

Figure 2. Simulation results, uneven - uneven distribution of resources, even - even distribution of resources, T[Erl] - traffic, Prej- the resulting probability of rejecting a service call

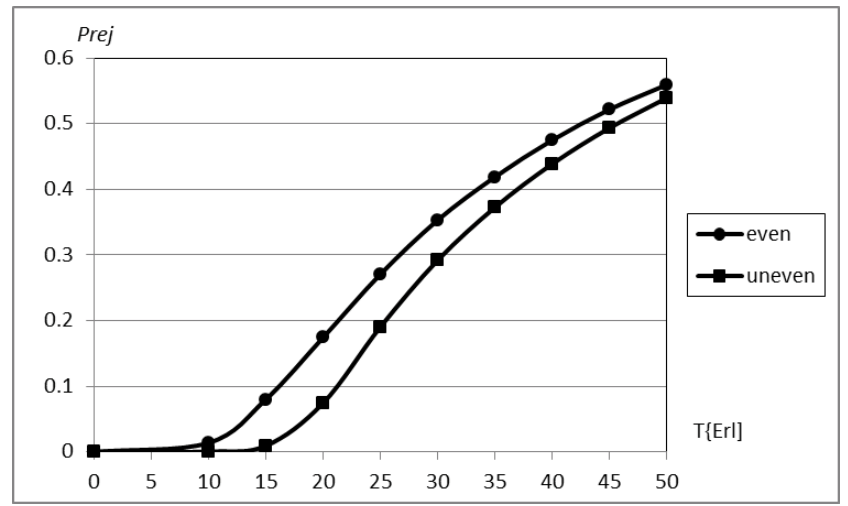

Figure 3. The result of the simulation test of the network with a diverse volume of traffic generated in the nodes

The provided results show that in both cases the introduction of the control of transmission resources causes the improvement of the transmission properties of an analysed structure.

The network was also tested for the case of a failure of any of the transmission connections. For this example, the connection marked with edge $c$ was selected. The change of the structure of the paths is shown in Table 12.

Table 12. The structure of minimum length paths in case of a failure of the $c$ edge

\begin{tabular}{|l|l|l|l|l|l|l|l|}
\hline Node & 0 & 1 & 2 & 3 & 4 & 5 & 6 \\
\hline 0 & - & $a$ & $b h$ & $a d$ & $a d i, b h f$ & $b k$ & $b$ \\
\hline 1 & $a$ & - & 0 & $d$ & $d i$ & $f$ & 0 \\
\hline 2 & $h b$ & 0 & - & $e$ & $g$ & $a b$ \\
\hline 3 & $d a$ & $\mathrm{~d}$ & $e$ & - & $i$ & $e g$ & $e h$ \\
\hline 4 & $a d i, b h f$ & $i d$ & $f$ & $i$ & - & $f g$ & $f h$ \\
\hline 5 & $k b$ & 0 & $g$ & $g e$ & $g f$ & $k$ & $k$ \\
\hline 6 & $b$ & $b a$ & $h$ & $h e$ & $h f$ & & - \\
\hline
\end{tabular}

By using the above way of conduct the result shown in Table 13 were obtained.

Table 13. The parameters describing the network in case of a failure of edge $c$

\begin{tabular}{|l|l|l|l|l|l|}
\hline$\sum_{n=1}^{N} H_{l n}$ & 0.800 & 1.200 & 0.000 & 0.800 & 1.000 \\
\hline$w_{\text {drcl }}$ & 0.097 & 0.145 & 0.000 & 0.097 & 0.121 \\
\hline$R E S_{l}$ & 31 & 47 & 0 & 31 & 39 \\
\hline$E d g e$ & $\mathrm{f}$ & $\mathrm{g}$ & $\mathrm{h}$ & $\mathrm{i}$ & $\mathrm{k}$ \\
\hline$\sum_{n=1}^{N} H_{l n}$ & 1.200 & 0.700 & 1.500 & 0.400 & 0.650 \\
\hline$w_{\text {drcl }}$ & & & & & \\
\hline$R E S_{l}$ & 0.145 & 0.085 & 0.182 & 0.048 & 0.079 \\
\hline
\end{tabular}


The chart (Figure 4) shows the result of the simulations.

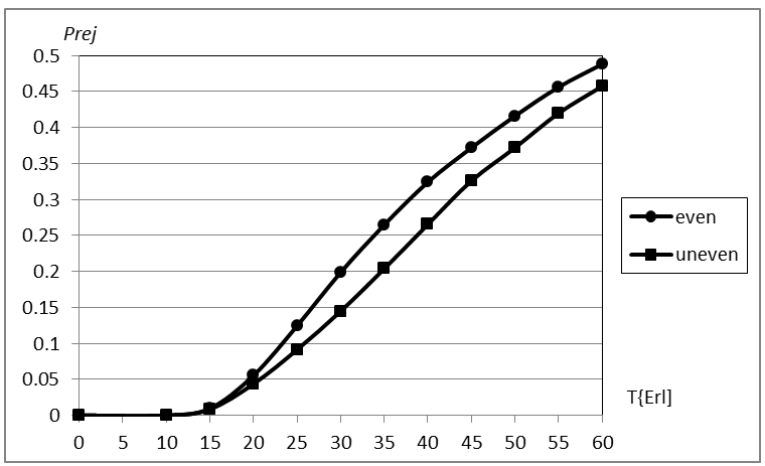

Figure 4. Simulation results

Also, in this case the introduction of the resource control makes it possible to more rationally utilise the transmission possibilities of the analysed network.

To check the extent to which the proposed modification influences more developed structures, the connection topology was studied. The topology is presented in Figure 5.

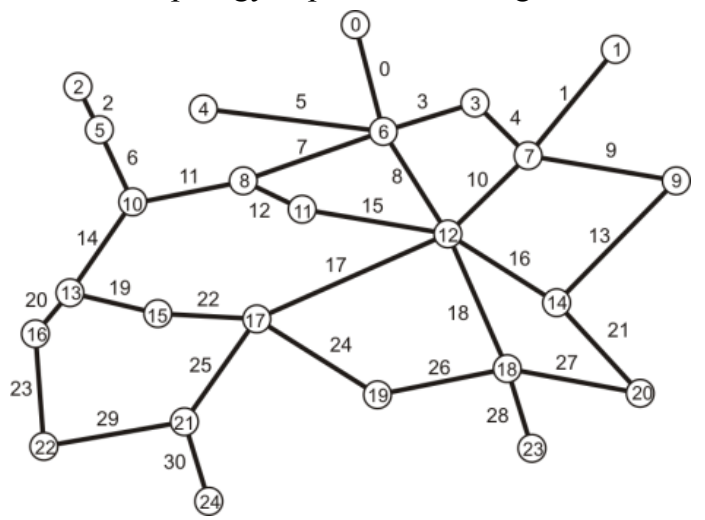

Figure 5. The graph describing the studied network

It consists of 25 nodes connected with 31 links. The distributions of traffic directed to network nodes were generated pseudo randomly (due to the size of the table they are not included in this paper). Table 14 shows the calculated values of usage of individual edges connecting the nodes of the network with the assumption that traffic generated in each node is the same.

Table 14. The table of calculated $H_{l n}$ coefficients

\begin{tabular}{|c|c|c|c|c|c|c|}
\hline Edge & 0 & 1 & 2 & 3 & 4 & 5 \\
\hline$\sum^{N} H_{l n}$ & 184.0 & 188.0 & 204.0 & 175.8 & 132.3 & 189.0 \\
\hline $\begin{array}{l}n=1 \\
\text { Edge }\end{array}$ & 6 & 7 & 8 & 9 & 10 & \begin{tabular}{|l|}
11 \\
\end{tabular} \\
\hline$\sum_{n=1}^{N} H_{l n}$ & 373.0 & 372.5 & 535.5 & 116.9 & 398.4 & 452.0 \\
\hline $\begin{array}{l}n=1 \\
\text { Edge }\end{array}$ & 12 & 13 & 14 & 15 & \begin{tabular}{|l|}
16 \\
\end{tabular} & \begin{tabular}{|l|}
17 \\
\end{tabular} \\
\hline$\sum_{n=1}^{N} H_{l n}$ & 205.1 & 111.8 & 299.0 & 286.5 & 291.2 & 642.7 \\
\hline $\begin{array}{l}n=1 \\
\text { Edge }\end{array}$ & 18 & 19 & 20 & 21 & 22 & 23 \\
\hline$\sum_{n=1}^{N} H_{l n}$ & 406.9 & 243.5 & 212.5 & 117.7 & 334.5 & 144.5 \\
\hline $\begin{array}{l}n=1 \\
\text { Edge }\end{array}$ & 24 & 25 & 26 & 27 & 28 & \begin{tabular}{|l|}
29 \\
\end{tabular} \\
\hline$\sum_{n=1}^{N} H_{l n}$ & 207.4 & 480.5 & 155.3 & 140.8 & 205.0 & 227.5 \\
\hline Edge & 30 & & & & & \\
\hline$\sum_{n=1}^{N} H_{l n}$ & 197.0 & & & & & \\
\hline
\end{tabular}


The table includes the calculated values of $w_{d r c l}$ coefficients and the distribution of resources $\left(R E S_{g}=3962\right)$.

Table 15. The distribution of transmission resources with the even distribution of the traffic generated in the network nodes

\begin{tabular}{|c|c|c|c|c|c|c|}
\hline Edge & 0 & 1 & 2 & 3 & 4 & 5 \\
\hline $\mathrm{W}_{\mathrm{drcl}}$ & 0.022 & 0.023 & 0.025 & 0.021 & 0.016 & 0.023 \\
\hline $\mathrm{RES}_{\mathrm{i}}$ & 348 & 364 & 396 & 332 & 252 & 364 \\
\hline Edge & 6 & 7 & 8 & 9 & 10 & 11 \\
\hline $\mathrm{W}_{\mathrm{drcl}}$ & 0.045 & 0.045 & 0.065 & 0.014 & 0.048 & 0.055 \\
\hline $\mathrm{RES}_{\mathrm{i}}$ & 716 & 716 & 1032 & 224 & 760 & 872 \\
\hline Edge & 12 & 13 & 14 & 15 & 16 & 17 \\
\hline $\mathrm{W}_{\mathrm{drcl}}$ & 0.025 & 0.014 & 0.036 & 0.035 & 0.035 & 0.078 \\
\hline $\mathrm{RES}_{\mathrm{i}}$ & 396 & 224 & 572 & 556 & 556 & 1240 \\
\hline Edge & 18 & 19 & 20 & 21 & 22 & 23 \\
\hline $\mathrm{W}_{\mathrm{drcl}}$ & 0.049 & 0.030 & 0.026 & 0.014 & 0.041 & 0.018 \\
\hline $\mathrm{RES}_{\mathrm{i}}$ & 776 & 476 & 412 & 224 & 652 & 284 \\
\hline Edge & 24 & 25 & 26 & 27 & 28 & 29 \\
\hline $\mathrm{W}_{\mathrm{drcl}}$ & 0.025 & 0.058 & 0.019 & 0.017 & 0.025 & 0.028 \\
\hline $\mathrm{RES}_{\mathrm{i}}$ & 396 & 920 & 300 & 268 & 396 & 444 \\
\hline Edge & 30 & & & & & \\
\hline $\mathrm{W}_{\mathrm{drcl}}$ & 0.024 & & & & & \\
\hline $\mathrm{RES}_{\mathrm{i}}$ & 380 & & & & & \\
\hline
\end{tabular}

The chart (Figure 6) includes the results of the simulations.

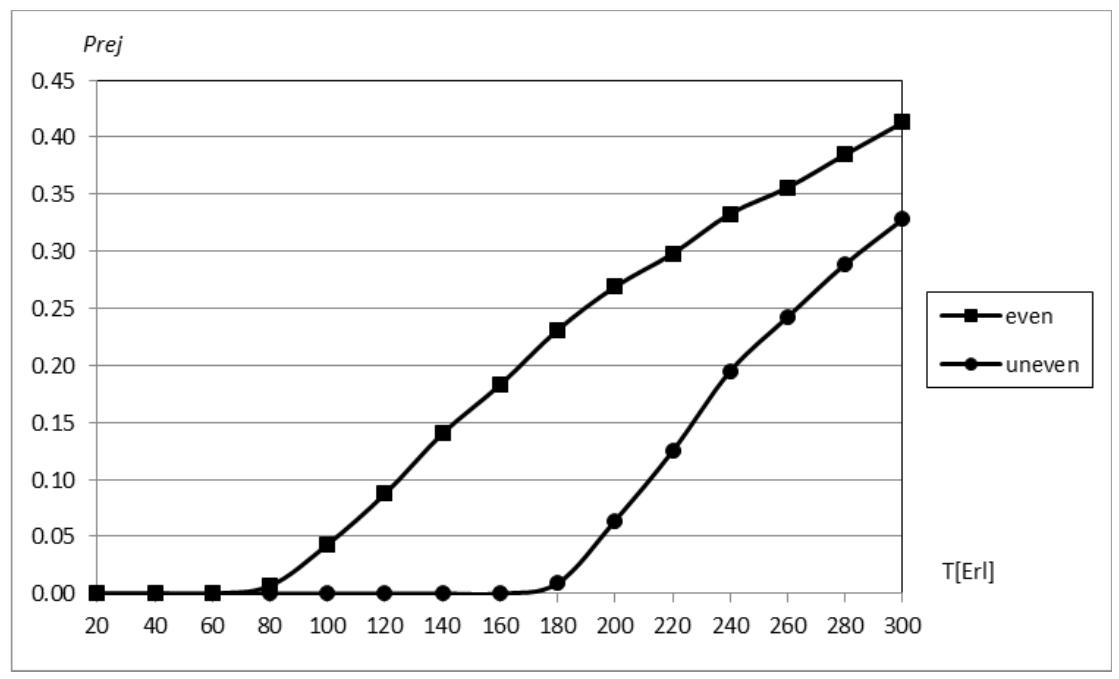

Figure 6. Results of simulations

The study of the analysed structure was repeated with the uneven distribution of the generated traffic. It was assumed that in nodes $1,8,24$ the traffic is two times larger, in nodes 11,22 - three times larger, 3, 6- four times larger, 4, 20 - five times larger, 14 - six times larger, 17 - seven times larger than in the remaining nodes.

Table 16 includes the results of the calculation with global resources $R E S_{g}=7928$.

Table 16. The distribution of transmission resources with the uneven distribution of the traffic generated in the nodes

\begin{tabular}{|l|l|l|l|l|l|l|}
\hline Edge & 0 & 1 & 2 & 3 & 4 & 5 \\
\hline$\sum_{n=1}^{N} H_{l n}$ & 280.0 & 413.0 & 353.0 & 471.3 & 355.9 & 740.0 \\
\hline $\mathrm{W}_{\text {drcl }}$ & 0.015 & 0.023 & 0.019 & 0.026 & 0.020 & 0.041 \\
\hline $\mathrm{RES}_{\mathrm{i}}$ & 350 & 366 & 396 & 334 & 254 & 366 \\
\hline Edge & 6 & 7 & 8 & 9 & 10 & 11 \\
\hline$\sum_{n=1}^{N} H_{l n}$ & 661.0 & 795.0 & 1480.0 & 213.3 & 784.1 & 894.0 \\
\hline $\mathrm{W}_{\text {drcl }}$ & 0.036 & 0.044 & 0.081 & 0.012 & 0.043 & 0.049 \\
\hline $\mathrm{RES}_{\mathrm{i}}$ & 714 & 714 & 1032 & 222 & 762 & 872 \\
\hline
\end{tabular}


Continuation of Table 16

\begin{tabular}{|c|c|c|c|c|c|c|}
\hline Edge & 12 & 13 & 14 & 15 & 16 & 17 \\
\hline$\sum_{n=1}^{N} H_{l n}$ & 420.4 & 227.7 & 553.0 & 669.0 & 945.6 & 1624.7 \\
\hline $\mathrm{W}_{\mathrm{drcl}}$ & 0.023 & 0.012 & 0.030 & 0.037 & 0.052 & 0.089 \\
\hline $\mathrm{RES}_{\mathrm{i}}$ & 396 & 222 & 572 & 556 & 556 & 1238 \\
\hline Edge & 18 & 19 & 20 & 21 & 22 & 23 \\
\hline$\sum_{n=1}^{N} H_{l n}$ & 809.2 & 438.0 & 371.0 & 387.4 & 654.0 & 260.0 \\
\hline $\mathrm{w}_{\mathrm{drcl}}$ & 0.044 & 0.024 & 0.020 & 0.021 & 0.036 & 0.014 \\
\hline $\mathrm{RES}_{\mathrm{i}}$ & 778 & 476 & 412 & 222 & 650 & 286 \\
\hline Edge & 24 & 25 & 26 & 27 & 28 & 29 \\
\hline$\sum_{n=1}^{N} H_{l n}$ & 346.4 & 1043.0 & 293.3 & 457.1 & 338.0 & 533.0 \\
\hline $\mathrm{W}_{\mathrm{drcl}}$ & 0.019 & 0.057 & 0.016 & 0.025 & 0.019 & 0.029 \\
\hline $\mathrm{RES}_{\mathrm{i}}$ & 396 & 920 & 302 & 270 & 396 & 444 \\
\hline Edge & 30 & & & & & \\
\hline$\sum_{n=1}^{N} H_{l n}$ & 408.0 & & & & & \\
\hline $\mathrm{W}_{\mathrm{drcl}}$ & 0.022 & & & & & \\
\hline $\mathrm{RES}_{\mathrm{i}}$ & 380 & & & & & \\
\hline
\end{tabular}

The results of the tests are presented in chart (Figure 7).

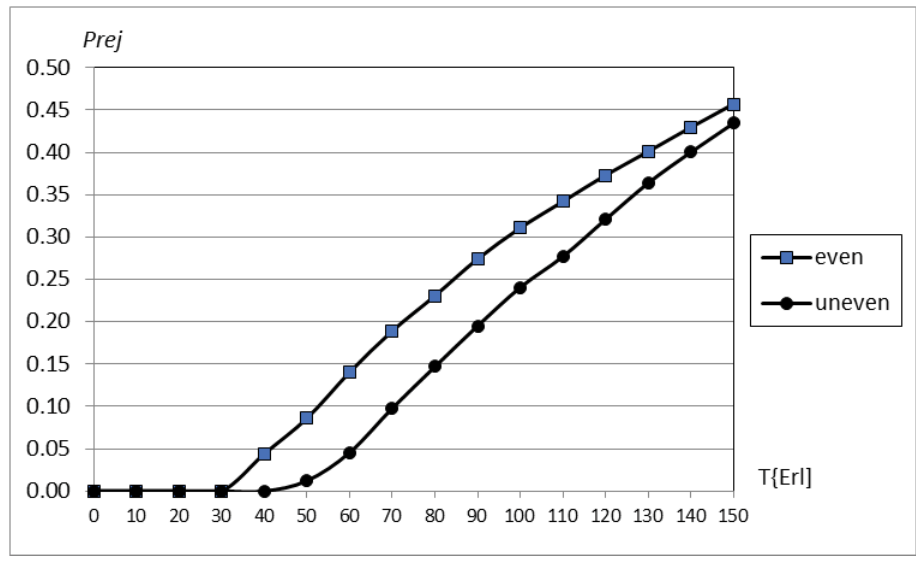

Figure 7. Results of simulations

\section{Summary}

The article presented the proposition to introduce the transmission resources control which would result in the improvement of communication properties of networks. To explain the procedure an example of a virtual network described with an irregular graph was used. A way of determining the traffic transmitted through individual graph edges corresponding to links connecting nodes of a network was discussed. On the grounds of the analysis of the results, the $w_{d r c}$ was defined which is used to calculate the distribution of resources assigned to edges in case of equal and diversifies volume of traffic generated in the nodes. The result of the calculations and analyses was the formulation of a software algorithm which prepares data necessary for simulation tests. They made it possible to check the influence of the proposed method of resource distribution on the transmission properties of the analysed networks. The conclusion from the results of the study is, that the introduction of the network resources management improves the transmission properties of the networks.

\section{Conclusions}

The aim of the conducted analyses and research was to improve the transmission properties of ICT networks. During the simulation tests of these networks, which were described by graphs, different utilisation of individual links (or more precisely - the transmission resources allocated to them) was found. 
This paper is theoretical but can be put to practice since, as it was proven in the article, if the structure of the network, the amount of traffic generated in nodes and the distribution of intermodal traffic are known, it is possible to assign proper transmission resources to individual links. It ensures more rational and efficient use of network, both during design and in use. The term 'transmission resources' means e.g. a number of timeslots or bandwidth used to transfer the usage information between network nodes.

In order to use the transmission capacity of the network more rationally, its global resources have been distributed between specific links in accordance with the values of the parameter called the unevenness coefficient determined for each edge. It was found that by introducing this modification, approximately one hundred percent utilisation of all resources intended for data transmission was achieved.

\section{References}

1. Bhuyan, L. N. (1987) Interconnection Networks for Parallel and Distributed Processing. IEEE Computer, 20(6), 9-12.

2. Bujnowski, S., Marciniak, T., Lutowski, Z., Marciniak, B., Bujnowski, D. (2017) Modeling Telecomunication Networks with the Use of Reference Graphs, Image Processing and Communications Challenges 9. IP\&C 2017. Advances in Intelligent Systems and Computing, 681, 115-126. Springer, Cham.

3. Bujnowski, S., Marciniak, T., Marciniak, B., Lutowski, Z., Marchewka, A. (2018) Analysis of the Influence of Transmission Resources Control in Tree Structure Networks. Image Processing \& Communication, 23(1), 11-19.

4. Bujnowski, S., Marciniak, T., Marciniak, B., Lutowski, Z. (2019) The Impact of Resource Control in Irregular Networks on Their Transmission Properties. JUCS - Journal of Universal Computer Science, 25(6), 591-611.

5. Chen, X., Li, J., Zhu, P., Tang, R., Chen, Z., He, Y. (2015) Fragmentation-Aware Routing and Spectrum Allocation Scheme Based on Distribution of Traffic Bandwidth in Elastic Optical Networks. Journal of Optical Communications and Networking, 7, 1064-1074, November 2015.

6. Coffman, K.G., Odlyzko, A.M. (2002) Growth of the Internet. Optical Fiber Telecommunicaitons IV B: Systems and Impairments. Academic Press, 2002, pp. 17-56.

7. Dai, Q., Zou, J., Shou, G., Hu, Y., Guo, Z. (2014) Network Virtualization Based Seamless Networking Scheme for Fiber-Wireless (FiWi) Networks. China Communications, 11, May 2014.

8. Deswal, S., Singhrova, A. (2012) Application Of Graph Theory in Communication Networks, International Journal of Application or Innovation in Engineering \& Management, 1(2), 66-70.

9. Diestel, R. (2001) Graph Theory. 4th Edition. Springer-Verlag, Heidelberg. Graduate Texts in Mathematics, Volume 173.

10. Graham, R., Knuth, D. and Patashnik, O. (1994) Concrete Mathematics. Addison-Wesley.

11. Heinzelman, W.R., Chandrakasan, A.P., Balakrishnan H. (2002) An application-specific protocol architecture for wireless micro sensornetworks. IEEE Transactions on Wireless Communications, $1(4), 60-70$.

12. Kaur, S., Mir, R.N. (2015) Quality of Service in WSN-A Review. International Journal of Computer Applications (0975 - 8887), 113(18).

13. Kotsis, G. (1992) Interconnection Topologies and Routing For Parallel Processing Systems. ACPC, Technical Report Series, ACPC/TR92-19.

14. Ledziński, D.; Śmigiel, S., Zabłudowski, Ł. (2017) Analyzing Methods Of Network Topologies Based On Chordal Rings. Turkish Journal of Electrical Engineering \& Computer Sciences, 25(6), 1-14.

15. Li, J., Wang, Y., Wu, Z., Feng, S., Qiu, X. (2017) A Prediction-based Dynamic Resource Management Approach for Network Virtualization, 13th International Conference on Network and Service Management (CNSM), November 2017.

16. Liu, J., Zhu, L., Sun, W., Hu, W. (2015) Scalable Application-Aware Resource Management in Software Defined Networking 2015, 17th International Conference on Transparent Optical Networks (ICTON), July 2015.

17. Mansourkiaie, F., Ahmed, M. H. (2016) Optimal and near-optimal cooperative routing and power allocation for collision minimization in wireless sensor networks, IEEE Sensors J., 16(5), 1398-1411.

18. Pedersen, J.M., Riaz, T.M., Dubalski, B., Madsen O.B. (2008) A Comparison Of Network Planning Strategies. 10th International Conference on Advanced Communication Technology, 702-707. ICACT.

19. Ramaswami, R., Sivarajan, K.N., Sasaki, G.H. (2010) Optical Network: A Practical Perspective. Ebook Burlington MA. Morgan/Kaufmann Elesevier. 
20. Tanaka, T., Inui, T., Kadohata, A., Imajuku, W., Hirano, A. (2016) Multiperiod IP-Over-Elastic Network Reconfiguration with Adaptive Bandwidth Resizing and Modulation. Journal of Optical Communications and Networking, 8, July 2016.

21. Wang, R., Bidkar, S., Meng, F., Nejabati, R., Simeonidou, D. (2019) Load-Aware Nonlinearity Estimation for Elastic Optical Network Resource Optimization and Management. Journal of Optical Communications and Networking, 11(5), May 2019.

22. Junming, Xu. (2010) Topological Structure and Analysis of Interconnection Networks. Springer Publishing Company, Incorporated 2010, ISBN 978-1-4757-3387-7. 